\title{
Archéopages Archéopages
}

Archéologie et société

Hors-série 3 | 2012

Nouveaux champs de la recherche archéologique

\section{Habitat et architecture au Néolithique : l'apport décisif de l'archéologie préventive}

Anaïck Samzun

\section{(2) OpenEdition}

9 Journals

Édition électronique

URL : https://journals.openedition.org/archeopages/479

DOI : 10.4000/archeopages.479

ISSN : 2269-9872

Éditeur

INRAP - Institut national de recherches archéologiques préventives

Édition imprimée

Date de publication : 1 janvier 2012

Pagination : 97-101

ISSN : 1622-8545

\section{Référence électronique}

Anaïck Samzun, « Habitat et architecture au Néolithique : l'apport décisif de l'archéologie préventive », Archéopages [En ligne], Hors-série 3 | 2012, mis en ligne le 01 janvier 2012, consulté le 23 février 2023. URL : http://journals.openedition.org/archeopages/479; DOI : https://doi.org/10.4000/archeopages. 479 
faire appel à d'autres champs de compétences pour nous éclairer, si possible, sur les usages de ces céramiques et les pratiques qui en découlent. Dans cette optique, des analyses chimiques ont été réalisées ${ }^{\mathbf{3}}$. Aucun caramel ou dépôt nétait visible, les résidus de matière amorphe ont été absorbés dans la matrice poreuse de la céramique. La première analyse chimique, réalisée dans le fond interne du micro-vase, a montré qu'il avait contenu une graisse d'origine animale (probablement de ruminant) ; sa transformation chimique indique des chauffages successifs, et un brai ${ }^{4}$ fabriqué à partir de bois de conifère. Une seconde analyse, réalisée sur le réceptacle central de la coupe à socle a montré qu'elle avait contenu différents matériaux organiques. Il s'agit de graisse animale, similaire au micro-vase, et d'une matière grasse d'origine végétale : une huile d'oléastre probablement, ainsi que des traces de résines de conifères ayant subi plusieurs chauffages, et conduisant à la formation d'hydrocarbures poly-aromatiques. Il serait tentant de voir dans l'association de ces deux objets un brûle-parfum et sa réserve. Toutefois, les composés organiques du brai détectés dans le micro-vase et ceux des résines de conifères ne sont pas identiques. Ces résidus ont cependant bien été chauffés, étant donnés les marqueurs de dégradation thermique (hydrocarbures polyaromatiques). Ainsi, si l'étude de ces résidus organiques ne permet pas d'élucider toutes nos interrogations, elle livre des renseignements novateurs sur la gestion des ressources naturelles par les populations du passé, et fournit également, associée à une étude typo-morphologique, des informations inédites sur l'usage, certainement cérémoniel, de céramiques découvertes en contexte funéraire.

\section{Habitat et architecture au Néolithique: l'apport décisif del'archéologie préventive}

Anaïck Samzun

Inrap, UMR 8215 « Trajectoires »

3 Par le Laboratoire

Nicolas Garnier, 63270 Vic-le-Comte. 4. La présence d'acides diterpéniques méthylés et d'hydrocarbures poly-aromatiques écarte l'hypothèse de résine brute, en faveur d'un brai: goudron végétal obtenu par pyrogénation de bois. contribué à renouveler les connaissances et les problématiques liées au Néolithique, ainsi qu'à développer des thématiques relatives à l'organisation des villages, à la fonction de certaines architectures et ont permis d'établir des parallèles avec des occupations contemporaines ailleurs en Europe.

\section{Une appréhension nouvelle des sites} néolithiques. L'approche et les méthodologies de l'archéologie préventive sont sensiblement différentes de l'archéologie programmée, dont les travaux se déroulent généralement pendant plusieurs courtes campagnes annuelles, dans des secteurs limités, dans des contextes environnementaux sélectionnés, et qui ont prévalu pendant une large partie du $\mathrm{xx}^{\mathrm{e}}$ siècle. En revanche, l'accroissement spectaculaire des interventions en archéologie préventive, diagnostics et fouilles, qui sont systématiquement mécanisées, réalisées sur de très larges superficies (parfois plusieurs dizaines d'hectares), dans le cadre d'aménagements extrêmement divers, qu'ils aient lieu en contexte de carrières, d'opérations autoroutières, ferroviaires (les lignes de TGV, LGV...), de canaux (le canal Nord), et même dans des zones urbaines (SaintDenis, Évreux) induisent un changement radical d'échelle pour l'appréhension des sites archéologiques. Ces recherches sont donc entreprises dans tous types de contextes géographiques et géomorphologiques, vallées, versants, plaines et terrasses alluviales, plateaux, zones humides : elles ne privilégient pas un secteur a priori propice, riche en vestiges ou révélé par la carte archéologique, puisque les fouilles préventives liées aux aménagements peuvent finalement concerner tous les terrains et tous les types de zones géographiques. Grâce aux décapages extensifs, elles ont ainsi permis de mettre au jour non seulement des habitats denses et riches en mobilier correspondant à des hameaux ou des villages parfois intégralement préservés et dégagés, des enceintes délimitant des espaces bâtis, mais également de très nombreuses occupations plus modestes (structures éparses, fosses, amas de débitage et aires d'activités diverses...) qui n'en sont pas moins dignes d'intérêt, en particulier quand elles sont attribuables à des étapes peu documentées du Néolithique.

Certaines étapes du Néolithique étaient en effet, jusqu'aux années 200o, très peu étudiées en raison de l'absence même de sites d'habitat, ou reconnues seulement dans des contextes funéraires et ce, sur de larges portions du territoire français. Le Néolithique ancien, qu'il s'agisse du Rubané (vers 5 200-5 000 av. notre ère), puis surtout de la culture de Blicquy-Villeneuve-Saint-Germain (B/VSG), entre 4.900 et 4700 av. notre ère, est en effet assez bien documenté avec, en particulier, l'apparition des toutes premières architectures en terre et en bois reconnues sur notre territoire, qui sont assez aisément décelables lors des opérations de diagnostic et ont été révélées sur de nombreux sites de la moitié nord de la France. En revanche, la situation est très différente pour les étapes moyenne, récente et finale du Néolithique, des étapes du Néolithique sur l'ensemble du territoir français. J. Dubouloz rappelle à son tour que la moitié nord de la France a été pendant longtemps considérée comme un no man's land documentaire pour toute cette période (Dubouloz, 2008). Notre objectif n'est pas ici de proposer un bilan exhaustif des découvertes d'habitats ou d'architectures néolithiques réalisées ces toutes dernières années en archéologie préventive, mais de rendre compte, au travers de quelques études de cas, d'avancées spectaculaires dans la détection, les approches méthodologiques, les études et les interprétations des vestiges mis au jour. Ces progrès ont largement 

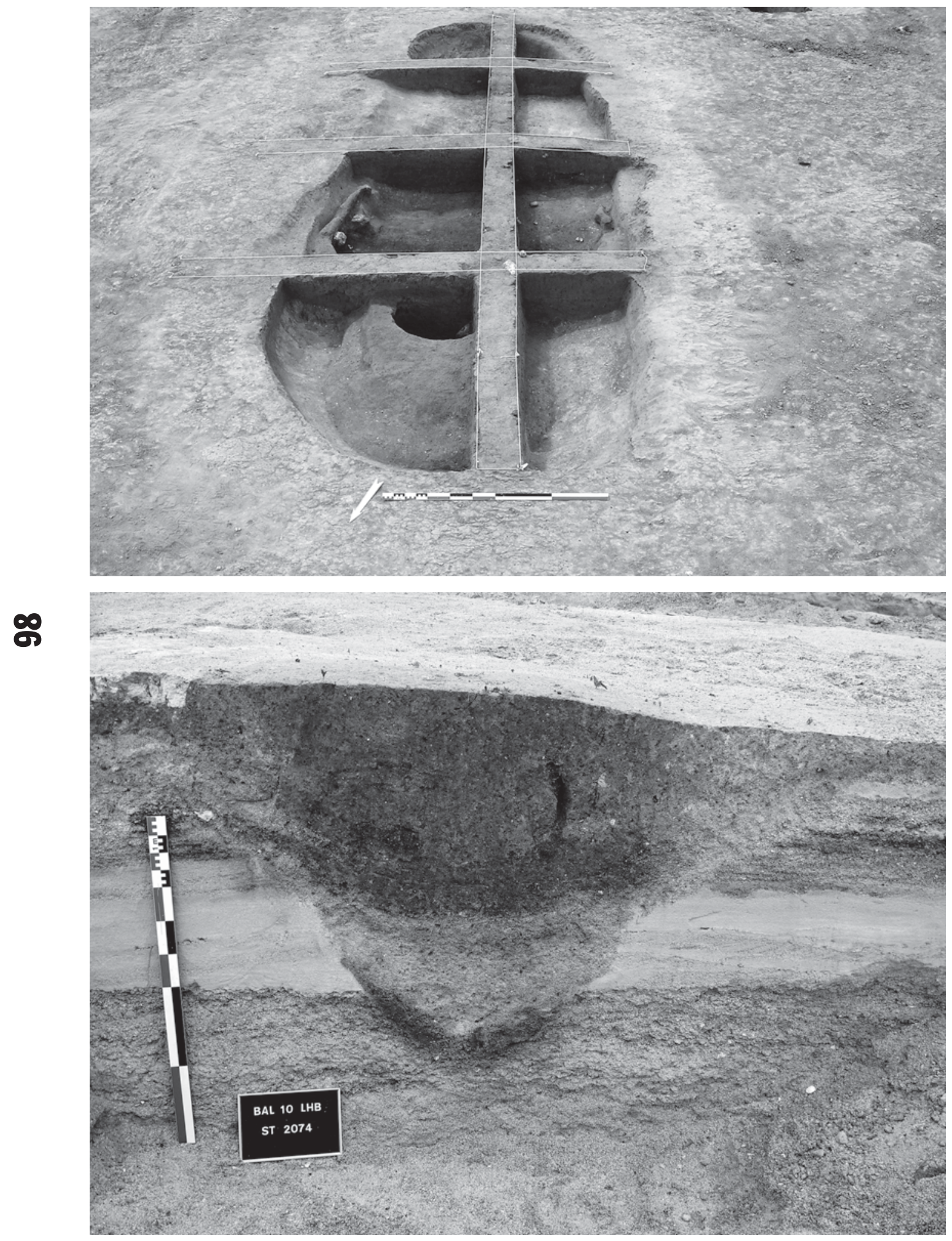

[Fig.1] Buthiers-Boulancourt (Seine-etMarne) : four creusé en sape dans le limon. Villeneuve-Saint-Germain récent (4900-4700 avant notre ère).
[Fig.2] Balloy " La Haute Borne"

(Seine-et-Marne) : puisard retrouvé

à proximité d'une fosse latérale

de maison. Villeneuve-Saint-Germain ancien. 
une vaste tranche chronologique de plus de deux millénaires et demi (entre 4700 et 2000 av. notre ère), pour laquelle seuls quelques rares sites d'habitat disséminés sur l'ensemble du territoire français avaient été jusque là découverts.

Les apports sur le Néolithique ancien. En France, les premières recherches portant sur cette étape du Néolithique ont été conduites dans les vallées du bassin parisien à partir des années 1960-1970 (Demoule, 2007), avec la mise au jour des premières maisons danubiennes. Les architectures danubiennes, de plan quadrangulaire ou trapézoïdal, de 15 à $45 \mathrm{~m}$ de long, de largeur constante, 6 à 9 m, avec leur orientation généralement nord-ouest/sud-est, sont construites sur un même modèle : des fosses, qui ont servi à l'extraction des matériaux et à la préparation du torchis pour enduire les murs clayonnés, puis utilisées comme fosses dépotoirs par les habitants, parfois très riches en mobilier archéologique, bordent systématiquement les parois latérales des maisons.

C'est désormais dans presque toute la moitié nord de la France que ces maisons, et également des hameaux ou des villages, ont été découverts et intégralement fouillés par la suite. À Changis «Les Pétreaux », en Seine-et-Marne (Lanchon, 2009), un site du Rubané final du Bassin parisien (RFBP) a été fouillé dans le cadre d'une extension de carrière en vallée. Seules deux unités architecturales distantes de $15 \mathrm{~m}$ l'une de l'autre ont été reconnues et l'absence d'autres structures néolithiques aux alentours confirme que les limites de cet habitat, dont la superficie est de $1250 \mathrm{~m}^{2}$, ont bien été atteintes. Si on n'avait pas eu la possibilité d'explorer les alentours, nous ne connaîtrions évidemment pas les limites exactes de cette implantation.

Des régions comme la Normandie et la Bretagne se sont récemment enrichies de vestiges rubanés et B/VSG (Marcigny et al., 2010), permettant d'appréhender la néolithisation vers l'ouest, dans des secteurs géographiques jusque-là pratiquement vierges de données archéologiques pour cette époque. À Poses « Sur la Mare » (Eure) (Bostyn, $2003)$, en fond de vallée, tout un habitat composé d'au moins une dizaine de maisons, dont les fosses latérales renfermaient un abondant mobilier, enrichit également les connaissances très lacunaires dans ce secteur et constitue désormais un référentiel. Encore plus à l'ouest, en Bretagne, les travaux sur le site de Pluvignon à Betton (Ille-et-Vilaine) où, dans une vallée, sur 2 ha, ont été repérés les restes d'au moins 6 maisons avec leurs fosses et peut-être des silos, ont révélé l'occupation la plus occidentale de la culture B/VSG (Pailler, et al. 2008).

De même, en région Centre, une douzaine d'occupations attestant d'une implantation régionale du Néolithique ancien ont été récemment reconnues (Creusillet, Irribaria, 2008) et un site fouillé (Dupont et al., 2010) est attribué au VilleneuveSaint-Germain (VSG) ancien. Ces découvertes permettent de retracer la néolithisation dans cette partie de la France qui est également englobée dans l'extension de la civilisation danubienne.
Enfin, dans le Pas-de-Calais, Loison-sous-Lens (Praud et al., 2010) représente le premier témoignage d'une occupation VSG dans cette partie de la France et atteste de contacts avec les populations implantées sur le territoire correspondant à la Belgique.

La finesse des méthodes de fouille et des analyses spatiales des activités, le remontage des poteries permettent également de confirmer que certaines maisons sont contemporaines et de définir le phasage d'un site et la spécialisation de certaines architectures, ainsi que la répartition des activités entre différentes unités architecturales. Les fouilles de nombreux sites d'habitat précisent non seulement les architectures, leurs variantes dans les procédés de construction, mais mettent également au jour, associées aux habitats, diverses structures comme des fours, des silos et des puisards mal documentés pour les étapes anciennes du Néolithique.

À Buthiers-Boulancourt, dans le sud-ouest de la Seine-et-Marne, dans le cadre d'une extension de carrière de sable en contexte de plateau, les architectures datées de la fin du VSG étaient mal conservées. Cependant, à proximité immédiate des fosses latérales, la découverte de plusieurs structures liées à la combustion, avec notamment un four domestique [Fig.1] (Samzun, Warmé, 2008) creusé en sape dans le limon, contribue à mieux discerner les activités se déroulant à proximité des habitats. À Balloy « la Haute Borne », également en Seine-et-Marne, étaient associées à deux architectures danubiennes des structures profondes à proximité des fosses latérales, de type puisards [Fig.2] (Meunier, Samzun, 2010), permettant probablement de capter l'eau lors de l'édification des maisons. De même, à Luzancy, c'est un silo qui se trouvait dans l'espace interne d'une maison (Lanchon, communication personnelle).

Certaines fouilles s'attachent également à expliquer quelles sont parfois les raisons de difficultés de détection des sites d'habitat. Ainsi à Lieusaint « Centre commercial 2 » (Seine-et-Marne) (Durand et al., 2008), J. Durand insiste sur les questions de lisibilité très complexes des sites néolithiques en contexte de plateau pour des raisons d'ordre pédologique et taphonomique. Elle s'interroge sur l'apparente rareté des vestiges du Néolithique ancien en contexte de plateau. À Lieusaint, on trouve en effet des fosses bien préservées, renfermant un mobilier assez riche, mais qui sont pratiquement invisibles lors des décapages, car leur partie sommitale a été érodée ou perturbée par des aménagements postérieurs. Les trous de poteau correspondant aux bâtiments ont complètement disparu en raison de l'érosion ou bien correspondaient à des structures plus légères qui n'ont pas laissé de traces.

\section{Les avancées sur le Néolithique moyen I-II} (4 700-3 600 av. notre ère). Â partir de la première partie du Néolithique, correspondant à

l'enracinement des populations sur leur territoire, une grande diversité de cultures caractérisées par la céramique se met en place (cultures Cerny, Chasséenne, Michelsberg, groupe de Noyen, groupe de Balloy, etc.) et, cependant, force est de constater que le nombre de sites d'habitats repérés et fouillés

.


est paradoxalement bien moins important quà l'étape précédente du Néolithique. Bien souvent, le Néolithique moyen n'est représenté que par quelques fosses isolées renfermant un peu de mobilier.

À la très grande homogénéité de l'architecture danubienne, semble succéder une grande variabilité des architectures qui pourraient également correspondre à la diversification des cultures et à une rupture avec la civilisation danubienne.

Les rares plans de maisons connues pour le début du Néolithique moyen sont très divers, parfois sujets à discussion, et se partagent entre bâtiments de forme circulaires et architectures de plan quadrangulaire. Cependant, ces dernières années, quelques découvertes remarquables sont venues heureusement documenter le début du Néolithique moyen et permettent, au moins partiellement, de retracer l'évolution des l'architecture et de l'habitat à la suite des bâtiments danubiens.

C'est à Pont-sur-Seine, dans l'Yonne, que d'importants ensembles architecturaux datés du Néolithique moyen au Néolithique récent, ont été mis au jour ces toutes dernières années. Au moins quatre structures circulaires ont été repérées et fouillées par Vincent Desbrosse et son équipe. Les architectures ont une superficie d'environ $80 \mathrm{~m}^{2}$ et deux d'entre elles présentent un espace interne cloisonné par une ligne de poteaux. Leur datation se situe aux alentours de 4500 avant notre ère. La fonction de ce type d'architecture n'est pas encore déterminée. Est-elle religieuse, cérémonielle, collective ou s'agit-il d'une habitation?

À Vivoin « Le Parc » (Sarthe), un bâtiment sub-trapézoïdal long d'une vingtaine de mètres, analogue aux architectures danubiennes, mais à l'orientation sensiblement différente, (nord-nordouest), a été récemment mis au jour (Ghesquière, Marcigny, 2003). Il est formé de 42 trous de poteau et l'espace interne est subdivisé par trois tierces : les 2 premières sont renforcées au niveau de l'axe par 3 poteaux qui ont pu soit consolider la charpente, soit soutenir une plateforme en élévation. Les fosses latérales sont absentes, mais plusieurs foyers lui sont associés. Le bâtiment et les structures annexes ont livré un mobilier céramique et lithique assez abondant.

À Beaurieux, «La Plaine » (Aisne), le très vaste bâtiment de forme trapézoïdale est inédit et est également daté de la première étape du Néolithique moyen [cf. encadré ci-contre].

La fonction de toutes ces structures reste problématique mais, selon toute vraisemblance, à une morphologie correspondent une fonction et un rôle spécifique.

$[\ldots]$
Le bâtiment monumental de Beaurieux «LaPlaine»: bâtiment uniqueou évolution terminale du modèle danubien?

Caroline Colas

Inrap

e bâtiment monumental de Beaurieux «La Plaine» se trouve au sein d'une nécropole monumentale qui comprend deux à trois monuments funéraires appartenant à la culture du Cerny (4.700-4.300 av. notre ère), un monument funéraire du Michelsberg ancien (4700-4300 av. notre ère) ainsi que quatre sépultures allant du Michelsberg ancien au Michelsberg récent (4 000-3500 av. notre ère). Ce bâtiment a déjà fait l'objet d'une présentation, c'est pourquoi nous n'en rappellerons ici que les principaux éléments (Colas et al., 2008 ; Colas et al., 2010). Sa longueur minimale était de $80 \mathrm{~m}$, mais les dimensions initiales de l'édifice sont inconnues du fait de la destruction ancienne de sa partie ouest. Orienté est-ouest, cet édifice comprend 43 trous de poteaux organisés en onze tierces et 10 fosses pour la façade avant. Sa façade est (20 $\mathrm{m}$ de large), opposée à la façade ouest ( $6 \mathrm{~m}$ de large), lui donne une forme trapéziforme prononcée [fig. 1]. Le module des trous de fondation à poteau unique varie de 1,50 à $2 \mathrm{~m}$. Les fosses de la rangée centrale et de la façade occidentale sont les plus profondes [fig. 2 et 3]. L'ancrage des trous de poteaux de la façade avant s'avère, en revanche, moindre. L'organisation d'ensemble, les comblements et les dynamiques de remplissage similaires ont permis de rejeter l'hypothèse d'une construction en plusieurs parties; seul un poteau indique l'existence d'une reprise.

Comme c'est souvent le cas pour les bâtiments, les comblements des structures de fondation renfermaient peu de mobilier : les tessons (matériaux et décors) évoquent la culture du Cerny et l'existence de plusieurs fragments d'os nous a permis d'avoir recours aux datations isotopiques (carbone 14, méthode AMS). L'intervalle de $4800 a ̀ 4,400 \mathrm{av}$. notre ère fourni par ces dernières a confirmé la datation obtenue par le mobilier sans apporter davantage de précision, puisqu'il couvre l'intégralité de la fourchette chronologique attribuée au Cerny.

Deux « épandages », situés de part et d'autre de l'édifice (l'un à une dizaine de mètres au sud et l'autre à une cinquantaine au nord) ont également livré un ensemble homogène de mobilier daté du Cerny éponyme, tandis qu'aucune autre période n'est représentée à proximité de ce bâtiment.

Les données architecturales pour le Néolithique moyen I sont particulièrement ténues. Les rares plans de maisons connus sont très variables : on relève des bâtiments rectangulaires orientés nordsud par exempleà Vivoin dans la Sarthe (Marcigny, Ghesquière, 1999), des maisons trapéziformes estouest à Herblay dans le Val d'Oise (Valais, 1995), ainsi que des maisons rondes, notamment dans le centre de la France (Verjux, 2007) en rupture totale avec tout ce qui précède. L'attribution des trois bâtiments voisins de Berry-au-Bac « Le Vieux Tordoir », dans l'Aisne, au Cerny, ne semble pas plus 
pertinente, compte tenu des données nouvelles sur l'habitat du Néolithique final (Dubouloz et al., 1996). Bien qu'il semble proche des édifices danubiens plus anciens (Coudat, 1998) - forme trapézoïdale, orientation, tierces) et qu'il pourrait apparaître comme une évolution ultime de ces derniers, il s'en démarque par ses dimensions, par l'absence du couloir (deux tierces plus rapprochées) et des fosses latérales qui servaient à extraire les matériaux nécessaires à la fabrication des murs. L'absence des fosses de construction découle peut-être de l'absence de murs. En effet, aucune preuve archéologique ne permet, aujourd'hui, d'envisager leur existence. La très bonne conservation des trous de poteaux et l'existence des deux couches voisines - vraisemblables niveaux de sols - démontrent que l'érosion n'a guère de chance d'être responsable de la disparition des poteaux de parois ou de sablières basses. En l'absence de ces murs, il faut donc imaginer une construction ouverte. Le gabarit et la profondeur spectaculaires des trous de poteaux, notamment de toute la rangée centrale, sont en adéquation avec les dimensions générales et la distance importante de certaines travées ; ces éléments autorisent à imaginer l'existence d'un toit à double pente, semblable à ceux qui devaient recouvrir les maisons d'habitations antérieures. L'allure générale de l'édifice serait proche de celle des halles de marché médiévales.La longueur du bâtiment de Beaurieux fait aussi exception. Les seuls bâtiments de même dimension sont les grandes maisons post-rubanés contemporaines d'Allemagne ou les grandes maisons du groupe polonais de Brzesc Kujawski chronologiquement un peu plus récentes (Hampel, 1989). Mais ces constructions ont toujours des poteaux de parois ou une sablière basse, certaines n'ont d'ailleurs plus que les sablières basses, et leur organisation interne est très déstructurée.

On peut imaginer que l'interprétation très « libre » du modèle danubien provient de son implantation auprès d'une nécropole monumentale. Il est probable que le bâtiment de Beaurieux n'appartenait pas à la sphère domestique, mais plutôt à la sphère religieuse.

COLAS C. BAILLIEU M., NAZE Y., 2008 : « Un bâtiment monumental Cerny à Beaurieux «La Plaine » (Aisne) », Internéo, 7, p. 59-69.

Colas C. (dir), Allard P., Chartier M., Hachem L., Maigrot Y., 2010 : Beaurieux (Aisne) La Plaine, Un « bâtiment monumental » exceptionnel, Rapport final d'opération, Inrap, SRA Picardie, 92 p., 68 fig.

COUDART A., 1998 : Architecture et société néolithique. L'unité et la variance de la maison danubienne, DAF, 67, Paris, 240 p., 194 fig.

Dubouloz J., Farruggia J.-P., Robert B., 1996 : « Bâtiments néolithiques non-rubanés à Berry-au-Bac "Le Vieux Tordoir", Aisne : présentation préliminaire », Internéo, p. 51-69.

Hampel A., 1989 : Die Hausentwicklung im Mittelneolithikum Zntraleuropa, Universitätsforchungen zur Prähistorischen Archäologie 1, Bonn, Rudolf Habelt, 360 p, 68 fig.

MARCigny C., GHesquiere E., 1999 : « Un bâtiment néolithique moyen I dans la Sarthe », Bulletin de la Société Préhistorique française, t. 96, fasc. 2, p. 264-266.

VALAIS A., 1995 : « Deux bâtiments atypiques associés à du matériel Cerny (Herblay, Val d'Oise) ", in Billard C. (DIR), Evreux, Actes du $20 e$ colloque sur le Néolithique, 1993, Revue Archéologique de l'Ouest ( $7^{\mathrm{e}}$ supplément), p. 57-63.

VERJUX C., 2007 : « Les bâtiments circulaires du Néolithique moyen dans le Bassin parisien », in Agogue O., Leroy D., Verjux C. (DIR), Camps, enceintes et structures d'habitat néolithiques en France septentrionale, Actes du 24e Colloque interrégional sur le Néolithique, Orléans, 19-21 novembre 1999, Revue Archéologique du Centre de la France (2 $7^{\mathrm{e}}$ supplément), p. 209-216.
[...]

L'éclosion d'ouvrages monumentaux :

les enceintes. La société néolithique connaît d'importantes transformations que l'on peut notamment identifier avec l'éclosion d'ouvrages monumentaux, requérant une main d'œuvre nombreuse et une organisation collective ad hoc, notamment pour aménager des enceintes visibles qui marquent le paysage. Il s'agit en effet d'abattre des centaines d'arbres, de les déplacer et ériger les fûts en palissade. Le principe architectural de ces structures consiste à creuser un ou plusieurs fossés continus ou discontinus et une palissade de bois interne est édifiée sur un talus aménagé en utilisant les matériaux extraits des fossés. Les entrées correspondent à l'interruption du fossé et de la palissade, mais peuvent être également conçues en chicane ou en couloir. Ces travaux se sont probablement déroulés collectivement, sous la conduite d'un chef. Leurs fonctions sont encore sujettes à discussion : habitat? Déroulement de cérémonies? Parcage des troupeaux? Les enceintes ont pu également jouer un rôle défensif ou protecteur, puisque la néolithisation étant achevée, les groupes néolithiques s'approprient désormais pleinement un territoire ou un terroir.

Les enceintes sont connues dès la fin du Néolithique ancien, mais se développent surtout dans la deuxième partie du Néolithique moyen. Mais, si ces importantes structures sont relativement bien attestées pour ces périodes, peu d'entre elles avaient été intégralement décapées et fouillées jusqu'au développement des travaux d'archéologie préventive. De même, les vestiges découverts dans l'espace interne de ces constructions étaient généralement erratiques et ne paraissaient pas constituer des habitats pérennes. En Auvergne, toute une série d'occupations du Néolithique moyen II, trouvées dans des contextes géomorphologiques divers, ont été mises au jour [cf. article p 117]. Elles combinent enclos, bâtiments rectangulaires, parfois à deux nefs sur tranchées de fondation et poteaux centraux porteurs, cabanes, aires d'activités spécialisées, structures foyères, formant un tissu de hameaux habités entre 3900 et 3500 av. notre ère, et attestant d'une augmentation importante de la population. À Cuiry-les-Chaudardes [cf. encadré p 103], l'occupation Michelsberg est représentée par quatre bâtiments d'assez petit module dont l'espace interne n'est pas subdivisé.

Le Néolithique moyen correspond clairement à une augmentation significative de la population, à un changement radical de société dont la hiérarchisation est perceptible avec l'aménagement des enceintes et de quelques grands bâtiments. À Carvin (Pas-de-Calais) [cf. encadré p 105], une vaste enceinte à plusieurs fossés et palissades renfermant un mobilier abondant caractéristique du Néolithique moyen II a été quasi intégralement fouillée. Elle ceinture deux bâtiments à poteaux centraux supportant une faîtière, qui sont accompagnés de quelques fosses. Cet agencement pose la question de la fonction même de ce type d'ouvrage monumental. Carvin est l'un des rares exemples où des bâtiments de ce type ont été 
découverts à l'intérieur même d'une enceinte. Dans ces structures, on met généralement au jour quelques fosses isolées, rarement plus. La raison principale en est l'érosion qui fait disparaître les structures les moins profondes et les difficultés importantes de reconnaissance du plan des architectures.

Les bâtiments sur poteaux centraux sont caractéristiques du Néolithique moyen II. On les trouve associés ou non à des enceintes. On constate qu'ils sont attestés sur une large portion du territoire français, au sud comme au nord de la Loire. Ces récentes découvertes permettent d'ores et déjà d'établir des parallèles entre ces habitats et ces architectures. [...]

\section{Les bâtiments du Michelsberg de Cuiry-lès-Chaudardes}

Caroline Colas

Inrap

7 occupation Michelsberg se compose de quatre bâtiments accompagnés d'une sépulture et de quelques fosses [Fig.1] (Colas et al., à paraître). Trois autres bâtiments partiellement reconnus existent probablement. Les types de bâtiments, leur couverture, leur mobilier permettent de percevoir le caractère exceptionnel du site. La façade occidentale, composée de quatre (bât. 1, 2 et 4) ou six (bât. 3,5 et 7 ?) trous de poteaux, forme une abside tandis que la façade orientale, plus étroite, n'en possède que deux [Fig.2]. Leur longueur, autour d'une dizaine de mètres de long, est stable tandis que la largeur varie de 3 et $6 \mathrm{~m}$, pour le petit côté, et de 4 et $8 \mathrm{~m}$, pour le grand. On peut ainsi distinguer deux catégories d'après leurs proportions : les bâtiments larges, dont la grande largeur correspond à environ la moitié de la longueur, et les bâtiments étroits, où elle n'en représente que le tiers.

Le diamètre des trous de poteaux oscille entre 0,15 et $0,80 \mathrm{~m}$ et leur profondeur entre $0,06 \mathrm{~m}$ (pour les plus arasés) et o,30 m (pour les mieux préservés). La plupart ont conservé l'empreinte du poteau disparu (fantôme). Il s'agit donc d'avant-trous destinés à recevoir le pilier en bois. La lecture des plans révèle l'absence de poteaux dans l'espace interne. L'espacement entre les poteaux latéraux suggère des murs extérieurs composés de poteaux d'armature destinés probablement à recevoir un clayonnage et du torchis et à supporter le toit. L'absence de poteaux faîtiers pose, cependant, problème si on envisage un toit à double pente. Des calculs effectués par des chercheurs allemands, en tenant compte des contraintes liées aux vents, au poids de la neige ainsi qu'au poids d'une couverture en motte de gazon, proposent deux possibilités pour couvrir un espace d'une dizaine de mètres (Zeeb-Lanz, 2001) : les chevrons du toit reposent sur les murs et sur le sol ou bien les chevrons sont enfoncés à l'oblique dans le sol [Fig.3]. La poutre faîtière est, dans ce cas, ligaturée au niveau de l'entrecroisement des chevrons. Donc, à moins de supposer l'existence de poteaux centraux ou d'une tranchée de fondation externe aujourd'hui disparus, la charpente devait reposer directement sur les murs extérieurs.

De même, l'espace interne n'était pas divisé ou bien les cloisons et les aménagements secondaires ont disparu. Le bâtiment 3 échappe à ce modèle. Plus large, il possède davantage de poteaux au niveau de l'abside. Une structure centrale composée de 4 poteaux était peut-être destinée à diminuer la portance et soutenir le toit [Fig.2]. Le bâtiment 5 appartient vraisemblablement à cette même famille. Le nombre de poteaux et la forme de l'abside conservée permettent un rapprochement avec le bâtiment 3. De même, la structure 142 a finalement été considérée sur plan comme un probable bâtiment supplémentaire, construit sur le même modèle que le bâtiment 3, mais dont les poteaux externes auraient disparu (orientation similaire). Des traces de structures en creux, considérées comme non probantes sur le terrain, pourraient en fait être le fond très mal conservé des poteaux de l'abside. Les absides, localisées du côté occidental, sont peut-être à interpréter comme des pignons d'entrée protégeant des pluies dominantes et des vents froids, comme c'est le cas pour les maisons du Néolithique ancien. Excepté cet argument indirect, rien, dans les plans, ne permet de situer précisément les entrées.

Les aspects techniques des tessons permettent de les assimiler à ceux de la culture Michelsberg (4. 200-3500 av. notre ère). Plusieurs trous de poteaux contenaient également des éclats de silex, de la faune et même un fragment de meule. La rareté des structures autour des unités d'habitation nous fait exclure la possibilité de mobilier erratique piégé lors du rebouchage des trous de poteaux. La présence de fragments d'os dans presque tous les bâtiments a permis d'effectuer des datations radiocarbones et de vérifier la pertinence du rattachement. Les cinq analyses situent cette occupation au Michelsberg récent entre 3700 et 3400 av. notre ère à deux sigmas Le seul autre gisement de cette période dans l'Aisne se situe à 800 mètres à l'ouest aux « Fontinettes ». Il s'agit du seul autre habitat du Michelsberg « ouvert ». Tous les autres sites sont des enceintes et un monument funéraire, et datent du Michelsberg ancien (4, 200-4 000 av. notre ère) à deux sigmas. Le seul autre site d'habitat ouvert de cette période dans l'Aisne se situe à 800 mètres à l'ouest aux «Fontinettes». Tous les autres sites sont des enceintes et un monument funéraire, datés du Michelsberg ancien (4, 200-4, 0oo av. notre ère).

Outre le plan potentiel mal conservé d'un bâtiment aux «Fontinettes », les sites de Berryau-Bac et d'Osly-Courtil sont les seuls de la région à avoir fourni des plans de maisons. Le bâtiment de Berry-au-Bac "Le Vieux Tordoir" est proche de ceux de Cuiry par ses dimensions (14 m de long sur 4 m de large) mais il en différe par la présence d'un rangée centrale de poteaux et son orientation nord-est/sud-ouest. La présence ou non d'une abside 
Ces édifications (poteaux équarris de forme rectangulaire pour l'une des palissades [Fig.1] et maisons à une rangée axiale de poteaux porteurs) suggèrent une gestion rigoureuse et maîtrisée du travail du bois, ainsi que l'invention de techniques nouvelles pour l'assemblage des charpentes : tenon- mortaise et chevillage.

L'ensemble des structures a livré une grande quantité de mobilier (surtout lithique et céramique) qui place le site de Carvin dans le groupe chronoculturel de Spiere, groupe contemporain du Chasséen septentrional et du Michelsberg. Certains objets confirment l'existence de réseaux d'échanges : quelques outils lithiques sont en silex originaire des minières de Spiennes (Hainaut belge), ou encore un pendentif en fluorite provient de gisements dont les plus proches connus se situent aux alentours de Charleroi (Hainaut belge également). Dans les fossés, des zones de rejet [fig. 2] se distinguent des zones de dépôt où le mobilier a été « mis en scène » avec des céramiques souvent entières [fig. 3-4].

Outre la question de la durée d'utilisation pour ce type de site, se pose celle de sa fonction : simple habitat fortifié ou place centrale d'un territoire organisé en groupe de fermes et de villages?

$[\ldots]$

Les occupations de la fin du Néolithique.

Les dernières phases correspondant à la fin du Néolithique (3600-2 ooo av. notre ère) restaient jusque très récemment, parmi les plus mal connues dans toute la moitié nord de la France.

En effet, c'est principalement par l'étude des sépultures collectives qu'elles étaient appréhendées (donc par des fouilles souvent anciennes et un mobilier spécifique à ce type de structures). Nos connaissances sont également fondées sur des enceintes dans le centre et centreouest de la France, et ailleurs sur des occupations comprenant des fosses domestiques isolées, un peu de matériel piégé dans les couches de sédiment, ainsi qu'un corpus limité de mobilier ne permettant pas de se faire une idée exacte du mode vie des populations et des modalités de leur implantation.

Aussi, les différentes occupations de la fin $\mathrm{du} \mathrm{IV}{ }^{\mathrm{e}}$ millénaire av. notre ère de Pont-sur-Seine (Yonne) [cf. encadré p 109], fouillées sur quelques 8 hectares, avec ses trois enclos comprenant une douzaine de bâtiments qui sont peut-être des habitations et deux architectures monumentales exceptionnelles dont les fondations sont en pierre, constituent-t-elles une avancée importante dans la connaissance de cette période.

Peu après, à la fin du Néolithique apparaissent, sur tout le territoire, de grands bâtiments collectifs dépassant parfois les $100 \mathrm{~m}$ de longueur, tels Moulinssur Céphons (Indre-et-Loire) ou Pléchatel (Illeet-Vilaine). À Houplin-Ancoisne (Pas-de-Calais), un bâtiment dépasse les $40 \mathrm{~m}$ de longueur [cf. encadré p 111]. Ces dernières années, plusieurs architectures de ce type, mais de dimensions moins importantes, ont également été révélées pour la première fois dans le Nord de la France, dans des contextes variés, qu'il s'agisse de fonds de vallée et de plateaux limoneux, même si on constate une préférence pour les zones humides. Les bâtiments sont de plan rectangulaire, parfois terminés en abside ; leur orientation est souvent est-ouest, mais pas systématiquement, et ils peuvent être en aire ouverte ou entourés d'une enceinte. Dans le Sud-Ouest de la France, à Douchapt « Beauclair » (Dordogne), Pierrick Fouéré a mis au jour des longs bâtiments de $66 \mathrm{~m}$ et $50 \mathrm{~m}$ de longueur pour une largeur de $18 \mathrm{~m}$, de même orientation (est-ouest), divisés en 2 nefs par un alignement central de très gros poteaux placés à intervalles réguliers, tous les $9 \mathrm{~m}$, Ils sont partiellement ceinturés par un enclos en terre et semblent avoir été réalisés d'un seul tenant. Malgré ces variantes architecturales, ils s'inscrivent également dans une tradition d'architecture monumentale à l'instar des exemples cités précédemment. Outre ces architectures de grandes dimensions, quelques découvertes de sites d'habitats viennent corriger les irrégularités documentaires. Des structures plus légères, associant des aires d'activités spécialisées avec foyers à des trous de poteau et des fosses dépotoirs comme à Trémonteix (Puy-de-Dôme) [cf. encadré p 113] où des lambeaux de sol sont préservés. À Bédoin «Limon-Raspail » (Vaucluse) (Cauliez et al., 2011), un site de plein air en altitude (2 878-2 626 av. notre ère) comprend des vestiges de murs en terre crue et des torchis qui présentent des traces de négatifs de clayonnage, des silos, des structures de combustion et des fosses-dépotoirs. Tous ces sites contribuent à combler les hiatus importants pour la fin du Néolithique, tant dans le Sud que le Nord de la France.

En 2 oo9, nous déplorions le faible niveau de redondance des sites néolithiques mis au jour dans le cadre particulier et limité de l'île-de-France (Samzun et al.,2009), et ce constat aurait très bien pu être établi dans d'autres régions françaises au cas par cas. À l'échelle nationale, ces lacunes se comblent progressivement comme nous venons de le voir. La documentation produite par l'archéologie préventive pour l'époque néolithique est à ce jour absolument considérable. L'exploitation de ces données commence à peine, même si la publication de certains sites majeurs est déjà réalisée ou enclenchée. De nombreux autres sites d'habitat sont cependant encore largement inédits. Nous faisons donc face à un double défi : d'une part, il est nécessaire d'assimiler un important corpus documentaire de façon à élaborer des synthèses interrégionales et à définir le cadre chronologique et culturel de ces découvertes qui a largement évolué ces dernières années. D'autre part, il est impératif de tenter d'appréhender la variabilité des habitats mis au jour, notamment leur hiérarchisation, afin d'aborder les questions ayant trait à l'organisation des sociétés néolithiques et à leur évolution. C'est bien entendu le souhait que nous formulons pour les dix prochaines années de l'Inrap.

BOSTYN F. (DIR.), 2003 : Néolithique ancien en Haute-Normandie : le village Villeneuve-Saint-Germain de Poses « Sur la Mare» et les sites de la boucle de Vaudreuil, Société préhistorique française, t. 4, travaux 4, 343 p. 
Cauliez, J., Blaise E., Bressy, Convertini F., Gilabert C., Hamon C., Lazard N., Negroni S., Ollivier V., Pelissier M., Petrequin, P., Piatschek C., Provenzano N., Renault S., 2011 : « Le site du Limon-Raspail à Bédoin dans le Vaucluse et le Néolithique final de moyenne vallée du Rhône », Bulletin de la Société préhistorique française, t. $108, \mathrm{n}^{\circ}$ 2, p. 263-330.

Creusillet M.-F., IrRibaria R., 2008: « Données récentes sur le VilleneuveSaint-Germain du sud-ouest du Bassin parisien », in BuRNEZ-LANOTTE L., Ilett M., Allard P., Fin des traditions danubiennes dans le Néolithique du Bassin parisien et de la Belgique (5100-470o av. J.-C.), Société préhistorique française, Mémoire XLIV, p. 161-18o.

Demoule J.-P., 2007 : « La révolution néolithique », Paris, La Découverte.

Dubouloz J., 2008 : « Beaucoup de sable et un peu de terre noire», Archéopages, hors-série, p. 49-55.

Dupont F., Liagre J., IrRibaria R., 2010 : « Sours les Ouches (Eure-etLoir). Un site original du Villeneuve-Saint-Germain ancien en région centre », in Billard C., Legris M. (DIR.), Premiers Néolithiques de l'Ouest. Cultures, réseaux, échanges des premières sociétés néolithiques à leur expansion, p. 75-100.

Durand J., Bemilli C., Boitard-Bidault E., Cayol N., DietschSellami M.-F., Durand S., Fechner K., Maigrot Y., Praud I., 2008 : « Difficulté d'identification des sites du Néolithique ancien en contexte de plateau : l'exemple du site de Lieusaint "Centre Commercial 2" (Seine-et-Marne) », in Praud I., Giligny F. (DIr.), Internéo, 7, Société préhistorique française, p. 7-22

Ghesquiere E., MARCigny C., 2003 : «L'habitat du Néolithique moyen I de Vivoin » Le Parc » (Sarthe), Bulletin de la Société préhistorique française, t. $100, \mathrm{n}^{\circ} 3$, p. 533-573.

LANCHON Y. (DIR.), 2009: « Le site de Changis « Les Pétreaux » (Seineet-Marne), Revue archéologique d'Ile-de-France, $\mathrm{n}^{\circ}$ 1, p. 43-92.

Marcigny C., Ghesquiere E., Juhel L., Charraud F, 2010 : « Entre Néolithique ancien et Néolithique moyen en Normandie et dans les îles anglo-normandes », in Billard C., Legris M. (DIR.), Premiers Néolithiques de l'Ouest. Cultures, réseaux, échanges des premières sociétés néolithiques à leur expansion, p. 117-161.

Meunier K., SAmzun A. : 2010 « Découverte d'un site du VSG ancien à Balloy "La Haute Borne" (Seine-et-Marne). Présentation des structures ", in Praud I., Besse M. (DIR.), Internéo, 8, Paris, p. 35-46.

Pailler Y., Marchand G., Blanchet S., Guyodo J., Hamon G., 2008: « Le Villeneuve-Saint-Germain dans la péninsule Armoricaine : les débuts d'une enquête ", in Burnez-Lanotte L., Ilett M., Allard P., Fin des traditions danubiennes dans le Néolithique du Bassin parisien et de la Belgique (5100-470o av. J.-C.), Société préhistorique française, Mémoire, XLIV, p. 91-111.

Praud I., Bostyn F., Cayol N., Hamon C., Ladureau, Lanchon Y., PinARD E., 2010 : « Entre Blicquy et Villeneuve-Saint-Germain. Présentation de la fouille d'un habitat du Néolithique ancien à Loison-sous-Lens (Pas-de-Calais) ». in BillaAd C., LEgris M. (DIR.), Premiers Néolithiques de l'Ouest. Cultures, réseaux, échanges des premières sociétés néolithiques à leur expansion, Presses universitaires de Rennes (coll. Archéologie et culture), p. 305-323.

Samzun, A., Cottiaux, R. LANCHON Y., 2009: « Néolithique en Ile-deFrance : la révolution reste à faire ", Archéopages, hors-série, p. 124-126.

SAMZUN, A., WARMÉ N., 2008 : « Fours, foyers et structures de combustion au Néolithique ancien et Néolithique moyen I : l'exemple du site de Buthiers-Boulancourt (Seine-et-Marne) », in Praud I., Giligny F. (DIR.), Internéo, 7, Société préhistorique française, p. 31-46.

\section{Une occupation du Néolithique récent à Pont-sur-Seine}

\author{
Vincent Desbrosse \\ Inrap \\ Virginie Peltier \\ Inrap
}

L' habitat de la fin du IV ${ }^{\mathrm{e}}$ millénaire est mal connu dans le bassin parisien. En effet il est généralement identifié à partir de mobilier piégé dans des horizons limoneux ou par des structures éparses ; ces traces limitées ne permettent donc pas de réfléchir sur les types d'occupation (Salanova, 2004, p. 78). La découverte d'un site d'habitat structuré de cette période, avec des plans de bâtiments préservés, revêt donc un intérêt tout particulier.

À Pont-sur-Seine (Aube), Le Haut de Launoy, l'organisation de l'occupation du Néolithique récent, est encore perceptible grâce à la préservation des fossés dans lesquels étaient enchâssés les poteaux de bois des palissades. Trois grands ensembles se dégagent. Un premier enclos de 1,4 ha renferme des bâtiments, certains étant à usage d'habitation [Fig.1]. L'agencement des constructions dans cette aire se précisera avec le résultat des datations en cours, mais on peut d'ores et déjà noter que la palissade dicte l'orientation de certains bâtiments d'habitation à proximité du fossé. Le deuxième enclos, d'une superficie de o,4 ha, renferme les deux constructions monumentales [Fig.2] ; leurs dimensions, les matériaux employés, le soin apporté à leur réalisation révèlent le caractère exceptionnel de ces deux édifices. Enfin, le dernier vaste ensemble regroupe la douzaine de bâtiments retrouvés hors des enceintes, principalement à l'est et au sud. La préservation de ces plans de maisons, pour des époques où ils font généralement défaut, est l'une des autres particularités du site de Pont-sur-Seine.

Jusqu'à présent, dans le bassin parisien, un seul plan de bâtiment de la deuxième moitié du $\mathrm{IV}^{\mathrm{e}}$ millénaire était connu. Fouillé sur les marges occidentales de l'Argonne à l'occasion des opérations d'archéologie préventive de la LGV Est, le site de Dampierre-le-Château (Marne), Liévaux, a livré les traces d'une habitation; elle a été datée de cette période à partir des charbons de bois contenus dans ses poteaux (Dugois et al., 2002, p. 10). En fournissant une vingtaine de plans de bâtiments, la fouille de Pont-sur-Seine contribue à combler le déficit d'informations. Mais, en l'absence de mobilier caractéristique, il a fallu recourir à des analyses au carbone 14 pour dater ces constructions. Les fondations des habitations dépassent rarement la vingtaine de centimètres sous le décapage. Avec une érosion plus forte ou dans des conditions d'observation moins favorables, seuls les fossés des palissades et les fondations des constructions monumentales auraient été retrouvés. L'enceinte la plus vaste aurait donc été « vide», cas de figure courant dans les enceintes contemporaines du Centre-Ouest; les fouilles récentes menées dans ce secteur (Ard et al., 2009) semblent remettre ce point en question. La faible profondeur des creusements des bâtiments n'est pas nécessairement liée à un fort arasement des vestiges. En effet sur le site de Dampierre-le-Château, où l'impact de l'érosion a été jugé faible, voire inexistant, la profondeur moyenne des poteaux est de 9,3 $\mathrm{cm}$ pour une paroi, et de 12,9 $\mathrm{cm}$ pour l'autre, sous 30 à 40 $\mathrm{cm}$ de terre végétale. L'axe faîtier est quant à lui un peu plus profond avec une moyenne de $27,4 \mathrm{~cm}$ (Dugois et al., 2002, p. 9).

L'occupation du Haut de Launoy est aussi caractérisée par la quasi-absence de fosses contenant un mobilier détritique du Néolithique récent. En revanche, des artefacts ont été piégés dans des niveaux limoneux sur le pourtour du site. Le recours à une mécanisation réfléchie a permis d'accroitre le volume de sédiments traité et donc de palier en partie la faible densité de mobilier. Néanmoins, une série de datations absolues sur du matériel sélectionné, issu de creusements, s'avère indispensable pour démêler l'écheveau de la chronologie. Enfin, et pour terminer par l'aspect 